\title{
Spectral measurements to probe the magneto-optical properties of commonly used organic dyes
}

\author{
Ward Brullot, Stefaan Vandendriessche and Thierry Verbiest \\ Laboratory for Molecular Electronics and Photonics, Division of Molecular Imaging and Photonics, \\ Department of Chemistry, Katholieke Universiteit Leuven, Celestijnenlaans 200D, Box 2425, 3001 \\ Heverlee (Leuven), Belgium
}

\begin{abstract}
Magneto-optically active organic compounds are of great technological interest. In contrast to inorganic materials, used in most current applications, organic materials have the advantages of fast response times, easy processing, low cost and abundant resources of starting materials available. Conjugated organic molecules or polymers have been reported to be eligible candidates for exhibiting magneto-optical responses. As part of an ongoing search in our group for efficient magneto-optical materials, eight organic conjugated dyes were screened for their Faraday rotation responses. Based on the obtained preliminary results, it is expected that planar, efficiently pi- conjugated molecules have a higher chance for exhibiting significant magneto-optical responses. Further research will be undertaken to confirm this hypothesis.
\end{abstract}

Keywords: Faraday rotation, magneto-optical spectroscopy, organic materials, organic dyes

\section{INTRODUCTION}

The Faraday effect or rotation, discovered in 1845 by Michael Faraday, ${ }^{1}$ is a magneto-optical phenomenon whereby the plane of polarization of a light beam is rotated in presence of a magnetic field along the propagation direction. A visual representation of Faraday rotation is shown in Figure 1. Empirically, the angle of rotation $\theta$ is given by ${ }^{2-4}$

$$
\theta=V B L
$$

with $\theta$ the angle of rotation in degrees $\left(^{\circ}\right), B$ the magnetic flux density in the direction of propagation in Tesla, $L$ the thickness of the sample traversed by the propagating light beam in meter and $V$ the Verdet constant of the sample in $\circ \cdot T^{-1} \cdot m^{-1}$.

The Verdet constant is a material property which depends on incident wavelength, ambient temperature and refractive index of the material under study. ${ }^{3}$ As an example, the Verdet constants of diamagnetic silica glasses at room temperature with incident $632.8 \mathrm{~nm}$ laser light vary from 230 to $1548^{\circ} \cdot T^{-1} \cdot m^{-1}{ }^{3}$

Contrary to natural optical rotation, Faraday rotation is a non-reciprocal effect. In other words, multiple crossings through a Faraday active medium do not cancel out the rotation of polarization but augment it. This non-reciprocity allows to use Faraday rotation for the design of optical isolators, magnetic field sensors, switches and modulators., ${ }^{2,5-8}$

Currently most of the above applications are based on inorganic crystals doped with paramagnetic ions, such as Yttrium-Iron garnets. Organic materials exhibiting a large Faraday rotation response are very desirable due to, among others, fast response times, easy processing, low cost and abundant resources of starting materials available.

Faraday rotation responses of common organic materials, such as toluene, methanol and xylene have been both theoretically predicted and experimentally measured. These materials show low magneto-optical activity. ${ }^{6,8}$ Pi-conjugated polymers, as e.g. specifically designed poly(arylene ethynylene) in certain conformations, are among the few organic materials which have been reported to exhibit significant magneto-optical responses. ${ }^{5,7}$

Since pi-conjugated materials have shown to be eligible candidates for exhibiting significant magneto-optical responses, it is useful to perform a screening of a wide variety of pi-conjugated materials. Within this framework, we studied the optical and magneto-optical responses of eight organic, conjugated molecular dyes. This screening is part of an ongoing search in our research group for efficient Faraday rotation materials for magneto-optical applications.

Corresponding author: W.B. E-mail: ward.brullot@fys.kuleuven.be

Nonlinear Optics and Applications VI, edited by Benjamin J. Eggleton, Alexander L Gaeta, Neil G. Broderick, Proc. of SPIE Vol. 8434, 84341G - (c) 2012 SPIE - CCC code: 0277-786X/12/\$18 - doi: 10.1117/12.921861 


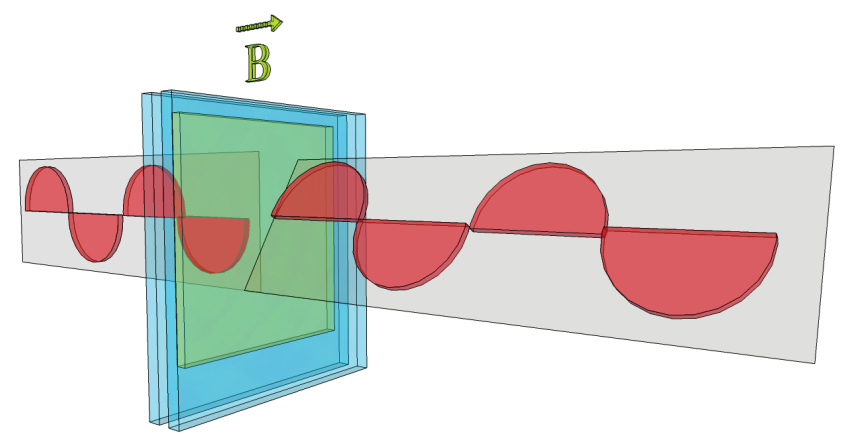

Figure 1. Representation of Faraday rotation. The plane of polarization of an incident light beam is rotated in presence of a magnetic field along the propagation direction.

\section{METHODS AND MATERIALS}

\subsection{Organic dyes and sample preparation}

For this work, eight conjugated organic dyes were screened for their Faraday rotation response in aqueous solutions. All studied molecules are shown in Figure 2.

The first three molecules, Acridine Orange (AO), Methylene Blue (MB) and Toluidine Blue (TB) are related heterocyclic aromatic compounds including three aromatic rings. While $\mathrm{AO}$ stems from the organic compound acridine, $\mathrm{MB}$ and $\mathrm{TB}$ are derived from a phenothiazine moiety. AO is mainly used as a cationic fluorescent dye for DNA or RNA detection or as a probe for measuring $\mathrm{pH}$ gradients across membranes. ${ }^{9-13} \mathrm{MB}$ has many uses including as a chemical redox indicator, as staining molecule in e.g. Wrights stain in biology and as a monoamine oxidase inhibitor antidepressant drug in medicine. ${ }^{10-14}$ TB finds application as a lignin stain test in plants or for identification of mast cells. ${ }^{10-15}$

1,1-diethyl-2,2-cyanine chloride (PIC) is a typical example of a cyanine dye used for studying surfaces through aggregation behavior and accompanied by changes in absorbance of fluorescence properties. It can also be used as a photochemical sensitizer. ${ }^{10-14,16}$

Molecules 5 and 6 on Figure 2, Crystal Violet (CV) and Malachite Green Carbinol (MGC) are structurally related. Possessing three phenyl moieties connected to a central carbon atom, they are also called triarylmethane dyes. CV is used as paper dye, as a component of print or pen ink and as stain in the biologically important Grams method for staining bacteria. Furthermore, the molecule exhibits medically relevant antibacterial, antifungal and antihelmintic properties. ${ }^{10-14,17,18}$ MCG is derived from Malachite Green, used as dyestuff for amongst others silk, leather and paper and in biology as a staining molecule. ${ }^{10-14}$

The last two molecules, Benzopurpurine 4B (BP) and Coomassie Brilliant Blue (CB) have extended molecular structures. BP consists of phenylazo-linked naphthalene derivatives, can be used as a $\mathrm{pH}$-indicator and is known for its chromonic properties due to the formation of large aggregates and liquid crystals at low concentrations. ${ }^{10-14,19} \mathrm{CB}$ is, like CV and MGC, a member of the triarylmethane dye family but features extended sidechains. The compound is mostly used in biochemical visualization protocols for proteins. ${ }^{10-14}$

Choice of studied compounds was based on the existence of a strong absorbance region in the UV-Visible region within the experimental range of our setups. Furthermore, the structural resemblance between molecules facilitates the identification of important structural characteristics for exhibiting Faraday rotation

Important to note is that the studied molecules are all prone to molecular aggregation in aqueous solutions. The aggregation states present in a sample can be probed by UV-Vis absorbance experiments, as aggregated molecules give rise to additional absorbance bands. Since the aggregation state might be an important factor in the generation of magnetooptical responses, it is necessary to take this parameter into account.

All dyes used in this work were obtained from Sigma-Aldrich and used as received without further purification or treatment. Aqueous solutions of the molecules were prepared by weighing the required mass of solid powder, quantitatively transferring the powder to a volumetric flask and adding MilliQ water. 
<smiles>CN(C)c1ccc2cc3ccc(N(C)C)cc3nc2c1</smiles><smiles>CN(C)c1ccc2nc3ccc(=[N+](C)[O-])cc-3sc2c1</smiles><smiles>Cc1cc2nc3ccc(=[N+](C)[O-])cc-3sc2cc1N</smiles><smiles>CCN1/C(=C/c2ccc3ccccc3[n+]2CC)C=Cc2ccccc21</smiles><smiles>CN(C)c1ccc(C(=C2C=CC(=[N+](C)[O-])C=C2)c2ccc(N(C)C)cc2)cc1</smiles><smiles>CN(C)c1ccc(C(O)(c2ccccc2)c2ccc(N(C)C)cc2)cc1</smiles><smiles>Nc1cc([N+](=O)[O-])c2ccccc2c1N</smiles><smiles>CNc1ccc(-c2ccc(/N=N/c3cc([O-])c4ccccc4c3N)c(C)c2)cc1C</smiles><smiles></smiles>

Figure 2. Chemical structures of the molecules studied in this work. 1) Acridine Orange (AO), 2) Methylene Blue (MB), 3) Toluidine Blue (TB), 4) 1,1-diethyl-2,2-cyanine chloride (PIC), 5) Crystal Violet (CV), 6) Malachite Green Carbinol (MCG), 7) Benzopurpurine 4B (BP) and 8) Coomassie Brilliant Blue R-250 (CB) 


\subsection{Spectral Faraday rotation and absorbance measurements}

Spectral Faraday rotation measurements were performed using a home-built setup. A free space EQ-99 Laser Driven Light Source (LDLS) from Energetiq inc. was used as the light source. The broadband white light beam was focused in a Newport VIS-NIR Cornerstone $2601 / 4$ m monochromator. Light exiting the monochromator then passed a polarizer and a photo-elastic modulator to obtain alternatively right-handed and left-handed circularly polarized light. This beam then passed through the sample holder of a cryogenic 1T DC magnet with the magnetic field vector parallel to the light propagation direction. After passing an analyzer, the light was detected by a photomultiplier tube. Due to a variety of specifically designed sample holders, the Faraday rotation setup is capable of handling a variety of thin film samples and solutions.

Standard spectral measurements spanned the 350-800nm UV-Visible range with data points taken every $10 \mathrm{~nm}$ and using 5 magnetic field strength values ranging from 0 to 0.6T. Adapted wavelength range, data point interval and the quantity of magnetic field strength values for which the Faraday rotation response was recorded were used as appropriate. For example, while investigating the Faraday rotation response of Methylene Blue in the absorbance region, a wavelength range from $500-750 \mathrm{~nm}$, a data point interval of $5 \mathrm{~nm}$ and 8 magnetic field strengths were used. To obtain a satisfactory signal-to-noise ratio and to avoid too low transmittance of light through the sample for detection on the Faraday rotation setup it was found that a maximum absorbance of the aqueous solutions of approximately 1 is optimal.

UV-Visible absorbance spectra of all samples were recorded on a Perkin-Elmer Lambda 900 spectrometer. Recorded spectra were analyzed in function of spectral purity of the compounds, possible molecular aggregation and a maximum absorbance value of approximately 1. Adjusting the maximum absorbance value was achieved by changes in molecular concentration or path length of the used cuvettes.

\section{RESULTS}

In this section, the results of UV-Vis and Faraday rotation measurements will be discussed. First the results for the individual molecules will be discussed and afterwards more general trends will be searched for.

Acridine orange, molecule 1 in Figure 2, was dissolved in water in two concentrations, namely $1 \times 10^{-4}$ and 1 $\times 10^{-5} \mathrm{~mol} / \mathrm{l}$. Absorbance spectra of both concentrations were measured and cuvette diameter was adjusted until an absorbance of approximately 1 was achieved. Both concentrations showed two absorption peaks in the UV-Vis region, one at approximately $495 \mathrm{~nm}$ and one around $465 \mathrm{~nm}$. The $495 \mathrm{~nm}$ peak can be attributed to the monomer, while the peak around $465 \mathrm{~nm}$ is due to the dimeric form of Acridine orange. ${ }^{9}$ The ratio of the maximum of the absorption peak at $495 \mathrm{~nm}$ to the maximum at $465 \mathrm{~nm}$ was found to be lower for the highest concentration, indicating the formation of more dimers at higher molecular concentrations.

Faraday rotation measurements on the $1 \times 10^{-5} \mathrm{~mol} / \mathrm{l}$ aqueous Acridine orange sample did not show a feature throughout the probed spectrum. The $1 \times 10^{-4} \mathrm{~mol} / \mathrm{l}$. solution, however, did show a small, broad peak spanning from $470 \mathrm{~nm}$ to $500 \mathrm{~nm}$, which means this peak is located within the high absorbance region of the Acridine orange monomer. Unfortunately, due to the steep rise of the absorbance in this wavelength range, which affects the sensitivity of our setup, the uncertainty on the obtained results is high. This mitigates the ability to draw firm conclusions from the data concerning this molecule.

Spectral UV-Visible absorbance and Faraday rotation results for Methylene Blue are shown in Figure 3. MB was dissolved in water in three concentrations, namely $1 \times 10^{-5}, 1 \times 10^{-4}$ and $5 \times 10^{-4} \mathrm{~mol} / \mathrm{l}$. Again, cuvette thickness was adjusted until an absorbance of one at the absorption peak was reached. The results shown in Figure 3 were obtained on the $5 \times 10^{-4} \mathrm{~mol} / \mathrm{l}$ solution sample. As can be seen, the absorbance spectrum shows two peak features, one around $665 \mathrm{~nm}$ and another around $605 \mathrm{~nm}$. The peak at approximately $665 \mathrm{~nm}$ can be attributed to monomer excitation, while the blue-shifted peak at around $605 \mathrm{~nm}$ is due to $\mathrm{H}$-aggregated or stacked molecules. ${ }^{20}$

Faraday rotation measurements revealed no apparent features for $1 \times 10^{-5} \mathrm{~mol} / 1 \mathrm{sample}$ but the $1 \times 10^{-4}$ and 5 $\times 10^{-4} \mathrm{~mol} / \mathrm{lsamples}$ did show peaks in the spectrum. As already stated, the results shown in Figure 3 are for the 5 $\times 10^{-4} \mathrm{~mol} / \mathrm{l}$ concentrated solution, as these results provided the best data for analysis. The Faraday rotation spectrum shown in Figure 3 exhibits two peak features, which show close resemblance to those observed in the absorbance spectrum. The peak maxima occur at the same wavelengths as for the absorbance peaks. The Faraday rotation signal within the absorbance peak regions was checked for artifacts due to a too steep increase in absorption or a too low transmission of the sample but no such artifacts could be detected. 


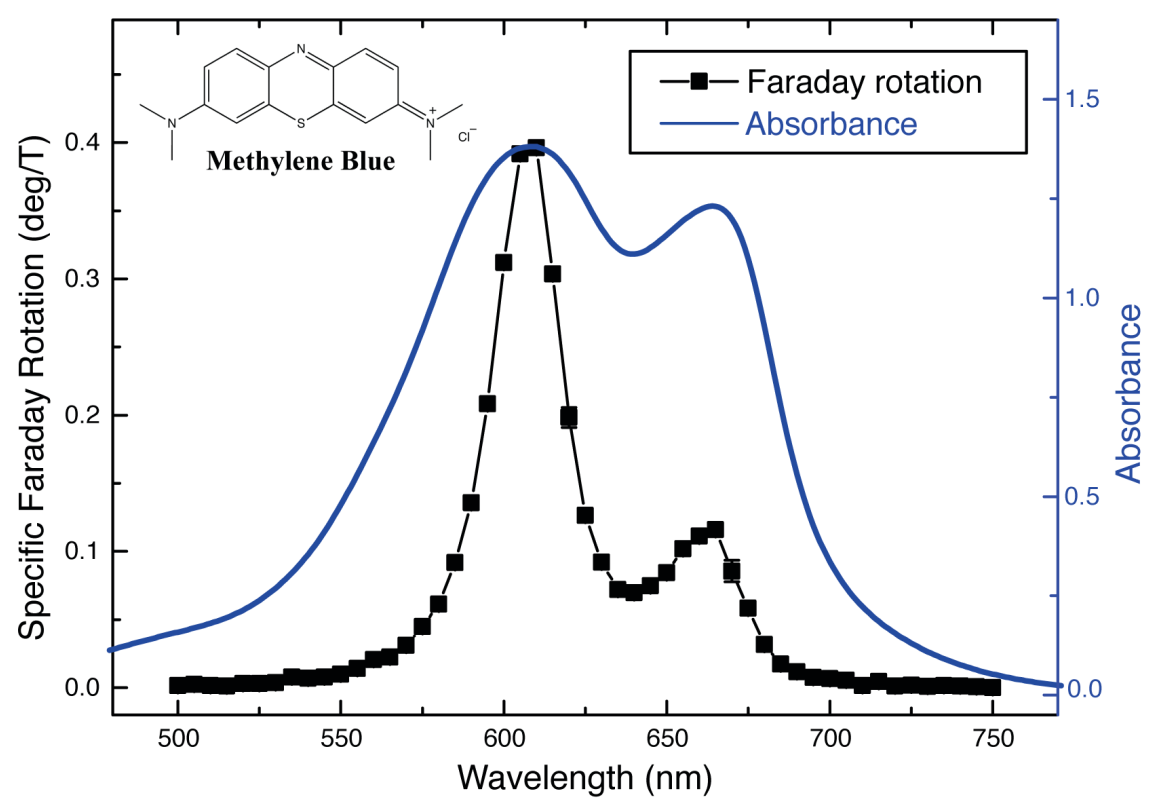

Figure 3. Faraday rotation (connected data points, left scale) and UV-Vis (solid blue line, right scale) data for a $5 \times 10^{-4} \mathrm{~mol} / 1 \mathrm{solution}$ of Methylene blue in water. Used cuvette thickness was $1 \mathrm{~mm}$. The Faraday rotation results show the same features as the UV-Visible absorbance data.

Despite the close resemblance, important differences could be noted between the peak features in the absorbance and Faraday rotation spectra. The ratio of the maxima of the $605 \mathrm{~nm}$ to the $665 \mathrm{~nm}$ peak is much higher for Faraday rotation than for absorption. This indicates that the dimeric form of Methylene Blue shows relatively more Faraday rotation than the monomer. An explanation for this observation could not yet be formulated but will be subject of further research. A second difference between the absorbance and Faraday rotation spectrum is that the full width at half maximum of the $605 \mathrm{~nm}$ peak is much smaller for Faraday rotation than for absorbance. While the origin of this observation remains unclear for the moment, it could be found in the great sensitivity of Faraday rotation for electronic resonances.

UV-Visible absorbance and Faraday rotation experiments for Toluidine blue, molecule 3 in Figure 2, were performed on three aqueous solutions of TB, namely $1.8 \times 10^{-4}, 1.8 \times 10^{-3}$ and $1.8 \times 10^{-2} \mathrm{~mol} / \mathrm{l}$. As the maximum of absorbance of the $1.8 \times 10^{-2} \mathrm{~mol} / \mathrm{l}$ sample was too high to be compensated for by reducing the cuvette thickness, this sample was discarded. Absorbance measurements on the other two samples revealed two peaks, one around $585 \mathrm{~nm}$ and another around $635 \mathrm{~nm}$. According to literature, the $635 \mathrm{~nm}$ peak is due to monomer excitation, while the $585 \mathrm{~nm}$ peak can be attributed to H-type or oblique angle aggregated molecules. ${ }^{15}$

Faraday rotation measurements on the TB samples proved very challenging, as the steep rise in absorbance around the peak regions caused major absorbance-related artifacts. Although the recorded Faraday rotation spectrum showed peak features in the absorbance peak regions for the molecules, the induced artifacts induced large uncertainties on the data, which rendered it impossible to draw conclusions on the existence of a magneto-optical response.

Molecule 4 of Figure 2, 1,1-diethyl-2,2-cyanine chloride (PIC), was dissolved in water in three different concentrations, namely $1 \times 10^{-5}, 1 \times 10^{-4}$ and $1 \times 10^{-3} \mathrm{~mol} / \mathrm{l}$. PIC has the known tendency to form J-aggregates, with red-shifted absorbance peaks, when dissolved in water at elevated concentrations. ${ }^{16}$ This was observed by the occurrence of two peaks in the UV-Visible absorbance spectra of all measured samples. The peak at approximately 490nm was attributed to monomer excitation, while the peak at $525 \mathrm{~nm}$ was caused by the presence of J-aggregated molecules. While both peaks were present in all samples, the ratio of the maxima of both peaks drastically changed upon increasing the concentration. The higher the concentration the higher the ratio of the $525 \mathrm{~nm}$ peak maximum to the $490 \mathrm{~nm}$ peak maximum, due to the formation of more J-aggregates. 
Results of spectral Faraday rotation measurements did not show any apparent features in the spectra for the prepared samples. Considering the range of concentrations measured, we can conclude that PIC does not display Faraday rotation that can be detected within the sensitivity range of our setup.

Crystal violet, molecule 5 of Figure 2, was dissolved in water in three concentrations, namely $1 \times 10^{-3}, 1 \times 10^{-4}$ and $1 \times 10^{-5} \mathrm{~mol} / \mathrm{l}$.Crystal violet molecules have the tendency to form $\mathrm{H}$-aggregates, causing blue shifted absorbance peaks, upon increasing concentration in aqueous solutions. This aggregation behavior was observed in the UV-Visible absorbance data of the prepared samples due to the presence of two absorbance peaks. The observed peak at around $588 \mathrm{~nm}$ is due to individual CV molecules and the peak at around 525nm due to aggregated CV molecules. ${ }^{17,18}$

The prepared sample with the highest concentration was not suitable for Faraday rotation measurements, as, even using a $1 \mathrm{~mm}$ cuvette, the absorption was too high for proper signal detection. Faraday rotation results for the other two samples showed no features in the probed spectrum. From this observation we can conclude that non-aggregated or slightly aggregated Crystal violet molecules do not exhibit measurable Faraday rotation.

Of molecule 6 of Figure 2,, Malachite green carbinol (MGC), one aqueous solution was prepared. The concentration of the sample was $1.6 \times 10^{-5} \mathrm{~mol} / \mathrm{l}$. Results of UV-Visible absorbance and Faraday rotation measurements on this sample are shown in Figure 4. The UV-Visible absorbance spectrum shows two absorption peaks, both from non-aggregated molecules. Important to note is that the maximum of absorption of MCG is somewhat red-shifted from that of Crystal violet. This shift can be attributed to the partial planarization of the substituted phenyl rings in MCG, increasing the degree of pi-electron conjugation. Crystal Violet, on the other hand, has a propellor-like structure, which allows a relatively lower degree of conjugation and consequently blue-shifted absorbance resonances.

As can be seen on Figure 4, the observed Faraday rotation is very small and shows quite some noise. The Faraday rotation signal within the absorption peak region was checked for artifacts due to a too steep increase in absorption or a too low transmission of the sample but no such artifacts were found. The results show a small, though not negligible Faraday rotation signal in the main absorption band. The peak in Faraday rotation located near the minor absorbance region around $525 \mathrm{~nm}$ could not be confirmed to be real. Important to note is that the maximum of Faraday rotation in the main absorption region occurs at a slightly shorter wavelength than the absorption maximum. The results obtained from this one solution will certainly be complemented by a more elaborate study of the origin of the magneto-optic response of this molecule in future research.

Of Benzopurpurine 4B, molecule 7 of Figure 2, two aqueous solutions were prepared with concentrations $1 \times 10^{-4}$ and $1 \times 10^{-5} \mathrm{~mol} / \mathrm{l}$. In agreement with literature, ${ }^{19}$ the UV-Vis spectrum showed two distinct absorption bands around $350 \mathrm{~nm}$ and $500 \mathrm{~nm}$. Upon increasing the concentration, the absorption coefficient of the 500nm absorption peak decreased, while the $350 \mathrm{~nm}$ band increased in intensity due to aggregation phenomena. ${ }^{19}$ From the UV-Visible absorbance data we can conclude that in the $1 \times 10^{-5} \mathrm{~mol} / \mathrm{l}$ sample very little aggregation has occurred, while in the $1 \times 10^{-4} \mathrm{~mol} / \mathrm{l}$ sample, aggregation is certainly present to some extent.

Faraday rotation measurements were performed on the samples, but no magneto-optical features were observed from these solutions. Consequently, we can conclude that neither single molecules, nor aggregated Benzopurpurine 4B molecules in the studied samples exhibit Faraday rotation measurable with our setup.

The eighth and last molecule on Figure 2, Coomassie Brilliant Blue R-250, was dissolved in three concentrations in water: $1.2 \times 10^{-3}, 1.2 \times 10^{-4}$ and $1.2 \times 10^{-5} \mathrm{~mol} / \mathrm{l}$. UV-Visible absorbance measurements showed that the sample with $1.2 \times 10^{-5} \mathrm{~mol} / \mathrm{l}$ concentration had a too high absorbance to be measured on the Faraday rotation setup. Absorbance measurements one the other samples showed one absorbance peak around $690 \mathrm{~nm}$ due to non-aggregated molecules, in agreement with literature. ${ }^{21}$

The Faraday rotation spectra obtained from the $1.2 \times 10^{-3}$ and $1.2 \times 10^{-4} \mathrm{~mol} / \mathrm{l} \mathrm{samples} \mathrm{did} \mathrm{not} \mathrm{exhibit} \mathrm{significant}$ features. It follows that non-aggregated Coomassie Brilliant Blue R-250 molecules do not show measurable Faraday rotation features within the probed wavelength range. This result is consistent with the absence of magneto-optical response of $\mathrm{CV}$ solutions, as $\mathrm{CB}$ is derived from the same triarylmethane moiety as $\mathrm{CV}$. As such $\mathrm{CB}$ assumes a similar conformation and thus intramolecular electronic pi-conjugation system as CV. 


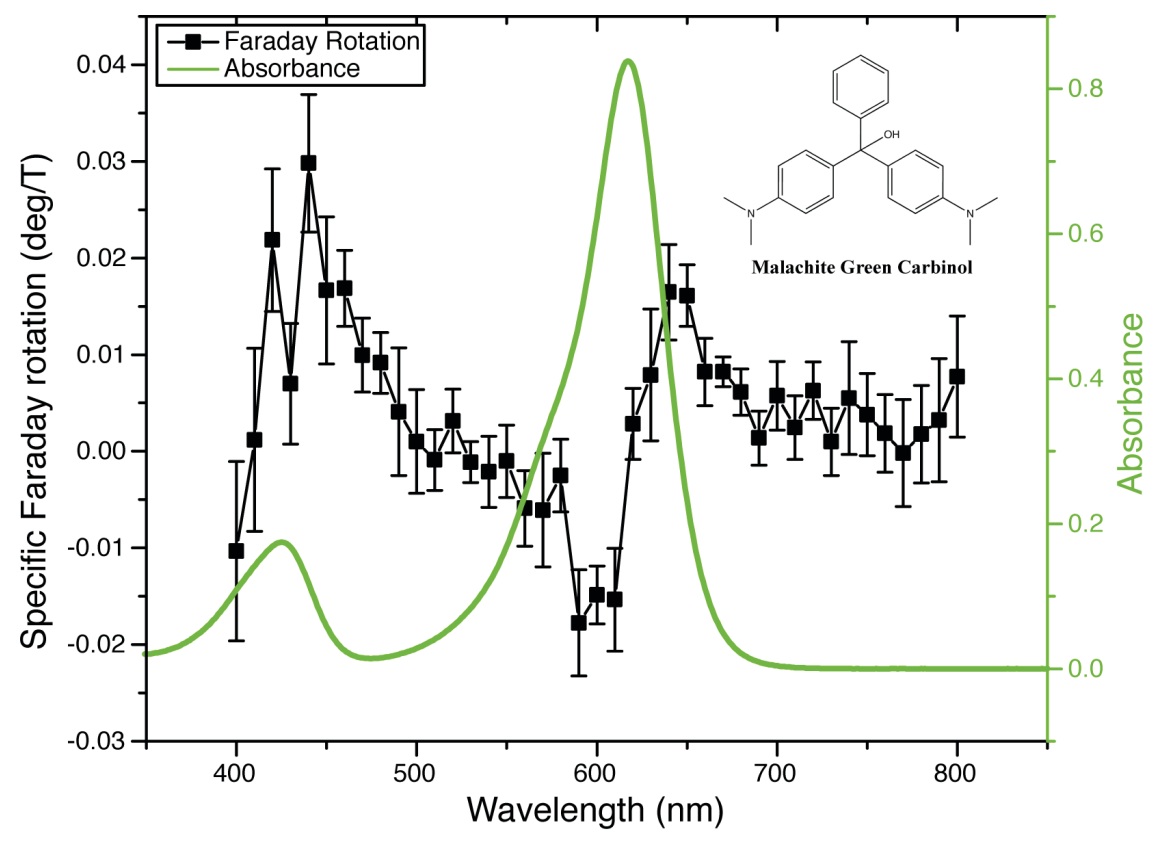

Figure 4. Faraday rotation (connected data points, left scale) and UV-Vis (solid green line, right scale) data for a $1.6 \times 10^{-5} \mathrm{~mol} / \mathrm{l} \mathrm{solution}$ of Malachite green carbinol in water. Used cuvette thickness was $2 \mathrm{~mm}$.

\section{DISCUSSION}

Of the eight studied molecules, two showed confirmed, measurable Faraday rotation features in the probed wavelength range. The strongest signal was observed for Methylene Blue, while a small effect was detected for Malachite green carbinol. Two other molecules, Acridine orange and Toluidine blue, possibly exhibit Faraday rotation, but could not be confirmed due to absorbance-related issues. For the other four molecules, namely PIC, Crystal violet, Benzopurpurine 4B and Coomassie brilliant blue R-250, no measurable magneto-optical signal could be detected.

Multiple insights can be gained from the obtained results. First of all, it is apparent that the family of heterocyclic aromatic compounds including three aromatic rings including Methylene blue, Toluidine blue and Acridine orange have shown the highest probability of exhibiting magneto- optical effects. This can be explained by the effective electron piconjugation in the planar heterocyclic aromatic ring system.

Secondly, within the family of triarylmethane dyes with Crystal violet, Malachite green carbinol and Coomassie Brilliant Blue R-250, only MGC shows a small magneto-optic feature in the probed wavelength range. As stated earlier in the results section, this result could also be explained by the more effective pi-conjugation in MGC by partial planarization as compared to $\mathrm{CV}$ and $\mathrm{CB}$, which retain more degrees of freedom.

In contrast to the planar or partly planar conjugated molecules, the structures that do not show any measurable Faraday rotation such as PIC, Benzopupurin 4B and Crystal violet, posses more degrees of freedom for intra-molecular torsions, rotations and stretch. This results in a less efficient pi-conjugation throughout the molecules, possibly explaining the absence of detectable magneto-optical features in the probed wavelength range.

Based on the insights gained and taking into account that more extensive research is necessary for confirmation, it seems that planar, efficiently pi-conjugated molecules have a higher chance for exhibiting Faraday rotation features in the probed wavelength range.

\section{CONCLUSIONS AND OUTLOOK}

In this work, eight organic, conjugated dyes were screened for their magneto-optical Faraday rotation response as part of an ongoing research effort for finding efficient Faraday rotation materials. Dyes were selected based on the existence of strong absorbance resonances in the UV-Visible region and mutual structural resemblances. 
From the obtained results, taking into account the fact that more molecules and variables need to be addressed, it could be concluded that planar, efficiently pi-conjugated molecules have a higher chance for exhibiting Faraday rotation features in the probed wavelength range.

Future work will include a systematic screening of conjugated organic molecules for their magneto-optical responses, so that structure-activity relationships might be uncovered. Many variables such as aggregation state, dipole moments, solvents and more will need to be addressed in order to establish the influence of these parameters on the magneto-optical response. Additionally, it has recently been demonstrated that organic conjugated dyes exhibit strong nonlinear optical response, ${ }^{22}$ at the second harmonic frequency. ${ }^{23}$ It would therefore also be interesting to probe the magneto-optical behavior of such molecules at the second harmonic. ${ }^{24}$ Indeed, when carefully employed, ${ }^{25}$ magnetization-induced second harmonic generation (MSHG) is highly sensitive to the magnetic moment in materials ${ }^{26}$ and can provide valuable complementary information to magneto-optical studies in the linear regime, such as Faraday rotation. Because MSHG requires high laser intensity, local field enhancements such as those provided by surface plasmons could be employed; ${ }^{27}$ whereby the organic conjugated dyes would either be bound to gold nanoparticles ${ }^{28}$ or placed in the hotspots of a nanopatterned gold surface. ${ }^{29}$ Similarly, local magnetic field enhancements could be provided by using magnetic materials, such as magnetic nanoparticles, ${ }^{2}$ magnetic-plasmonic core-shell nanoparticles ${ }^{30}$ or bilayers. ${ }^{31}$

\section{ACKNOWLEDGEMENTS}

The authors would like to thank V.K. Valev, M. Bloemen and M. Vanbel for useful discussions concerning the work and the publication. We acknowledge financial support from the Fund for scientific research Flanders (FWO-V), the Agency for Innovation by Science and Technology in Flanders (IWT) and the Catholic University of Leuven (GOA). W.B. is grateful for support from the IWT. S.V. is grateful to the FWO-Vlaanderen.

\section{REFERENCES}

[1] Faraday, M., [Experimental Researches in Electricity Vol. III], Bernard Quaritch, London (1855). pp. 1-12.

[2] Brullot, W., Reddy, N., Wouters, J., Valev, V., Goderis, B., Vermant, J. and Verbiest, T., "Versatile ferrofluids based on polyethylene glycol coated iron oxide nanoparticles," J. Magn. Magn. Mater. 324, 1919-1925 (2012).

[3] Valev, V. K., Wouters, J. and Verbiest, T., "Differential detection for measurements of Faraday rotation by means of ac magnetic fields," Eur. J. Phys. 29(5), 1099-1104 (2008).

[4] Valev, V. K., Wouters, J. and Verbiest, T., "Precise measurements of Faraday rotation using ac magnetic fields," Am. J. Phys. 76(7), 626 (2008).

[5] Araoka, F., Abe, M., Yamamoto, T. and Takezoe, H., "Large Faraday Rotation in a -Conjugated Poly(arylene ethynylene) Thin Film," Appl. Phys. Express 2, 011501 (2009).

[6] Botek, E., Champagne, B., Verbiest, T., Gangopadhyay, P. and Persoons, A., "A joint theoretical-experimental investigation of the Faraday effect in benzene, toluene, and p-xylene.," Chemphyschem 7, 1654-6 (2006).

[7] Koeckelberghs, G., Vangheluwe, M., Doorsselaere, K. V., Robijns, E., Persoons, A. and Verbiest, T., "Regioregularity in Poly(3-alkoxythiophene)s: Effects on the Faraday Rotation and Polymerization Mechanism," Macromol. Rapid Comm. 27, 1920-1925 (2006).

[8] Vandendriessche, S., Valev, V. K. and Verbiest, T., "Faraday rotation and its dispersion in the visible region for saturated organic liquids.," Phys. Chem. Chem. Phys. 14, 1860-4 (2012).

[9] Palmgren, M. G., "Acridine orange as a probe for measuring $\mathrm{pH}$ gradients across membranes: mechanism and limitations.," Anal. Biochem. 192, 316-21 (1991).

[10] Lide, D., [CRC Handbook of Chemistry \& Physics], CRC Press, Boca Raton, 74 ed. (1993).

[11] Horobin, R. W., Kiernan, J. A., and Conn, H. J., [Conn's biological stains: a handbook of dyes, stains and fluorochromes for use in biology and medicine ], BIOS (2002).

[12] Hunger, K., [Industrial dyes: chemistry, properties, applications], John Wiley \& Sons (2003).

[13] Sabnis, R. W., [Handbook of Biological Dyes and Stains: Synthesis and Industrial Applications], John Wiley \& Sons (2010).

[14] Healy, D., [The creation of psychopharmacology], Harvard University Press (2002).

[15] D'Ilario, L. and Martinelli, A., "Toluidine blue: aggregation properties and structural aspects," Model. Simul. Mater. Sc. 14, 581-595 (2006). 
[16] Würthner, F., Kaiser, T. E. and Saha-Möller, C. R., "J-aggregates: from serendipitous discovery to supramolecular engineering of functional dye materials.," Angew. Chem. Int. Edit. 50, 3376-410 (2011).

[17] Stork, W., Lippits, G. and Mandel, M., "Association of crystal violet in aqueous solutions," J. Phys. Chem. 76(12), 1772-1775 (1972).

[18] Lueck, H., Rice, B. and McHale, J., "Aggregation of triphenylmethane dyes in aqueous solution: dimerization and trimerization of crystal violet and ethyl violet," Spectrochim. Acta A 48(6), 819-828 (1992).

[19] McKitterick, C. B., Erb-Satullo, N. L., LaRacuente, N. D., Dickinson, A. J. and Collings, P. J., "Aggregation properties of the chromonic liquid crystal benzopurpurin 4B.," J. Phys. Chem. B 114, 1888-96 (2010).

[20] Patil, K., Pawar, R. and Talap, P., "Self-aggregation of methylene blue in aqueous medium and aqueous solutions of Bu4NBr and urea," Phys. Chem. Chem. Phys. 2(19), 4313-4317 (2000).

[21] Chial, H., Thompson, H. and Splittgerber, A., "A spectral study of the charge forms of Coomassie Blue G," Anal. Biochem. 209, 258-266 (1993).

[22] De Meulenaere, E., Chen, W.-Q., Van Cleuvenbergen, S., Zheng, M.-L., Psilodimitrakopoulos, S., Paesen, R., Taymans, J.-M., Ameloot, M., Vanderleyden, J., Loza-Alvarez, P., Duan, X.-M. and Clays, K., "Molecular engineering of chromophores for combined second-harmonic and two-photon fluorescence in cellular imaging," Chem. Sci. 3(4), 984 (2012).

[23] Verbiest, T., Clays, K. and Rodriguez, V., [Second-order Nonlinear Optical Characterization Techniques: An Introduction ], CRC Press, Boca Raton, FL (2009).

[24] Kirilyuk, A., "Magnetization-induced-second-harmonic generation from surfaces and interfaces," JOSA B 22(1), 148-167 (2005).

[25] Valev, V. K., Gruyters, M., Kirilyuk, a. and Rasing, T., "Influence of quadratic contributions in magnetization-induced second harmonic generation studies of magnetization reversal," Phys. Status Solidi B 242, 3027-3031 (2005).

[26] Valev, V., Kirilyuk, A., Dalla Longa, F., Kohlhepp, J., Koopmans, B. and Rasing, T., "Observation of periodic oscillations in magnetization-induced second harmonic generation at the Mn/Co interface," Phys. Rev. B 75, 3-6 (2007).

[27] Brongersma, M. L. and Kik, P. G., [Surface Plasmon Nanophotonics], vol. 2010, Springer (2010).

[28] Nappa, J., Revillod, G., Abid, J.-P., Russier-Antoine, I., Jonin, C., Benichou, E., Girault, H. H. and Brevet, P. F., "Hyper-Rayleigh scattering of gold nanorods and their relationship with linear assemblies of gold nanospheres," Faraday Discuss. 125, 145 (2004).

[29] Valev, V. K., Silhanek, A. V., De Clercq, B., Gillijns, W., Jeyaram, Y., Zheng, X., Volskiy, V., Aktsipetrov, O. A., Vandenbosch, G. A. E., Ameloot, M., Moshchalkov, V. V. and Verbiest, T., "U-shaped switches for optical information processing at the nanoscale.," Small 7, 2573-6 (2011).

[30] Brullot, W., Valev, V. and Verbiest, T., "Magnetic-plasmonic nanoparticles for the life sciences: calculated optical properties of hybrid structures," Nanomed-Nanotechnol. (2011). In press. DOI:10.1016/j.nano.2011.09.004.

[31] Valev, V., Silhanek, A., Gillijns, W. and Jeyaram, Y., "Plasmons Reveal the Direction of Magnetization in Nickel Nanostructures," ACS nano 5(1), 91-96 (2011). 